\title{
Centrosomes: Methods for Preparation
}

Karin Habermann, Max Planck Institute for Molecular Genetics, Berlin, Germany

Bodo MH Lange, Max Planck Institute for Molecular Genetics, Berlin, Germany

Based in part on the previous version of this Encyclopedia of Life Sciences (ELS) article, Centrosomes: Methods for Preparation by Michel Bornens.

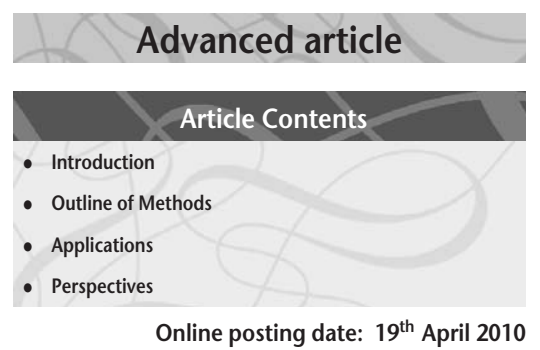

The centrosome of higher eukaryotic cells is the main microtubule-organising centre. To understand the molecular mechanisms underlying this organelle's biogenesis and important functions in several cellular processes, such as microtubule nucleation, cell division and stress response, it was critical to develop methods for isolating biochemically meaningful quantities of centrosomes. Centrosomes have been isolated from a variety of organisms and based on these preparations, numerous aspects of this intriguing organelle's morphological, functional and biochemical properties have been uncovered. Better isolation procedures along with the development of new technologies, like RNAi (ribonucleic acid interference) and the increasing accuracy of mass spectrometry and electron microscopy techniques, have profoundly improved our knowledge of the centrosome, leading to a better understanding of its implications in various cellular processes and in diseases.

\section{Introduction}

The centrosome is the primary microtubule-organising centre of higher eukaryotes. Its functions and components are highly conserved among divergent organisms. In most mammalian cells, the centrosome is composed of a pair of centrioles surrounded by an amorphous protein matrix, the pericentriolar material (PCM). The PCM contains proteins responsible for microtubule nucleation, like $\gamma$-tubulin, anchoring and scaffolding proteins and signalling

ELS subject area: Cell Biology

How to cite:

Habermann, Karin; and Lange, Bodo MH (April 2010) Centrosomes: Methods for Preparation. In: Encyclopedia of Life Sciences (ELS). John Wiley \& Sons, Ltd: Chichester.

DOI: 10.1002/9780470015902.a0002597.pub2 molecules. Centrioles generally consist of nine triplet microtubules which are arranged in a pinwheel-like structure. In fungi, plants and animals, centrioles also act as basal bodies, necessary for the outgrowth of cilia and flagella. In dividing cells, the centrosome is duplicated once per cell cycle such that at the onset of mitosis, a cell has two centrosomes, which serve as poles of the mitotic spindle. During cytokinesis, one centrosome is distributed to each daughter cell. See also: Cell Cycle; Centrosome Cycle; Cilia and Flagella; Mitosis; Tubulin and Microtubules

To understand the molecular mechanisms underlying this organelle's biogenesis and important functions in several cellular processes, like microtubule nucleation, cell division, stress response and cancer, it was critical to develop methods for isolating biochemically meaningful quantities of centrosomes. Such centrosome preparations, first obtained by Blackburn et al. (1978) from mammalian cells, have led to the determination of additional morphological features, molecular composition and function of this organelle. Various genetic, ribonucleic acid interference (RNAi) and biochemical approaches have contributed to the identification of centrosome components. Although the molecular make-up of this large protein complex is still not known in its entirety, significant advances have been made in the past decade regarding the identification of centrosome-associated proteins and their interactions, mainly due to improved isolation protocols and more sensitive mass spectrometry techniques in combination with the availability of complete genome sequences of an increasing number of model organisms. See also: Genome Sequencing; Mass Spectrometry in Biology; RNA Interference (RNAi) and MicroRNAs

\section{Outline of Methods}

\section{Sources}

Centrosomes have been isolated from a variety of sources, including cultured animal cells (Gosti-Testu et al., 1986; Bornens et al., 1987), animal tissue (Komesli et al., 1989), fly embryos (Moritz and Alberts, 1999; Lange et al., 2000), yeast (Rout and Kilmartin, 1990), green algae (Dutcher, 
1995; Keller and Marshall, 2008), slime moulds (Roobol et al., 1982; Gräf, 2001), surf clam oocytes (Palazzo and Vogel, 1999) and sea urchin zygotes (Thompson-Coffe et al., 1996). Overviews of isolation protocols are provided by Bornens and Moudjou (1999) and Kellogg et al. (1994); a summary of selected methods is given in Table 1.

\section{Cell lysis}

Most of the protocols involve a cell lysis step by mechanical disruption or detergent treatment in low ionic strength buffers to dissociate centrosomes from the nucleus. Pretreatment of cells with the microtubule depolymerising drug nocodazole is required to ensure disruption of centrosomal interactions with the cytoskeleton and to obtain highly enriched centrosomes from human lymphoblastic cells. However, such drug treatment can be omitted when isolating centrosomes from animal tissues such as thymus as low ionic strength buffer alone is sufficient to achieve effective dissociation of the centrosome from the nucleus and cytoskeleton. Furthermore, nuclease treatment of lysates is a beneficial step for reducing chromatin contamination. When other starting materials are used for centrosome isolation, alternative approaches are employed. For example, in Dictyostelium, the centrosome is tightly connected to the nucleus by a fibrous linkage which can be dissociated by lysis in the presence of pyrophosphate and shearing forces. Yeast spindle pole bodies, the functional analogues of higher eukaryotic centrosomes, are embedded in the nuclear membrane. Thus, purification is based on isolating centrosome-nucleus complexes that are further dissociated by mechanical shearing or by dispersion of the internal contents of the nuclei. The isolation of basal bodies from Chlamydomonas requires deflagellation of cells before lysis. Centrosomes have also been isolated from Drosophila embryos and Spisula solidissima oocytes. In both methods, the ease and cost efficiency of obtaining large quantities of starting material yielding large amounts of highly purified centrosomes present an important advantage over some of the other mentioned sources. See also: Algal Flagella; Dictyostelium: Cell Culture and Molecular Tools; Saccharomyces cerevisiae: Applications

\section{Isolation procedure}

Regardless of the source material used, virtually all isolation protocols for centrosomes, spindle pole bodies and basal bodies are based on the organelles' sedimentation properties. Here we provide an overview of the isolation procedure from tissue culture cells and outline the method for isolating centrosomes from early Drosophila embryos, which was described by Lange et al. (2000) and in more detail by Lehmann et al. (2006), and which, for the first time, employs an immunoaffinity purification step.

Lysates obtained from cultured mammalian cells are cleared from contaminating chromatin and other cell debris by sedimentation and filtering through nylon mesh. Centrosomes are concentrated by centrifuging the cleared lysates over a Ficoll or sucrose cushion followed by velocity sedimentation in a discontinuous sucrose gradient. The peak centrosome fraction is usually found at around $55 \%$

Table 1 Summary of centrosome isolation methods from different model organisms

\begin{tabular}{|c|c|c|c|c|}
\hline Source & Method & Yield $(\%)$ & $\begin{array}{l}\text { Enrichment } \\
\text { (fold) }\end{array}$ & Reference \\
\hline \multirow[t]{3}{*}{$\begin{array}{l}\text { Cultured } \\
\text { mammalian cells }\end{array}$} & $\begin{array}{l}\text { Ficoll cushion followed by one } \\
\text { sucrose step gradient }\end{array}$ & 30 & n.d. & $\begin{array}{l}\text { Mitchison and Kirschner } \\
\text { (1986) }\end{array}$ \\
\hline & One sucrose step gradient & 50 & 2000 & Bornens et al. (1987) \\
\hline & Ficoll cushion & $30-75$ & n.d. & $\begin{array}{l}\text { Blomberg-Wirschell and } \\
\text { Doxsey (1998) }\end{array}$ \\
\hline Calf thymus & One sucrose step gradient & 20 & n.d. & Komesli et al. (1989) \\
\hline Drosophila & One sucrose step gradient & 10 & 1000 & Moritz and Alberts (1999) \\
\hline embryo & $\begin{array}{l}\text { Two sucrose step gradients } \\
\text { followed by immunoisolation }\end{array}$ & n.d. & n.d. & Lehmann et al. (2006) \\
\hline $\begin{array}{l}\text { Saccharomyces } \\
\text { cerevisiae }\end{array}$ & $\begin{array}{l}\text { One sucrose step gradient } \\
\text { followed by continuous } \\
\text { Percoll-sucrose gradient }\end{array}$ & 45 & 600 & $\begin{array}{l}\text { Rout and Kilmartin } \\
(1990)\end{array}$ \\
\hline \multirow[t]{2}{*}{$\begin{array}{l}\text { Chlamydomonas } \\
\text { reinhardtii }\end{array}$} & $\begin{array}{l}\text { Two sucrose step gradients } \\
\text { followed by Percoll gradient }\end{array}$ & n.d. & n.d. & Dutcher (1995) \\
\hline & $\begin{array}{l}\text { Two sucrose step gradients } \\
\text { followed by continuous gradient } \\
\text { of Nycodenz }\end{array}$ & n.d. & n.d. & Kellogg et al. (1994) \\
\hline $\begin{array}{l}\text { Dictyostelium } \\
\text { discoideum }\end{array}$ & Two sucrose step gradients & 50 & 1000 & Gräf (2001) \\
\hline Spisula solidissima & One sucrose step gradient & n.d. & 3000 & Palazzo and Vogel (1999) \\
\hline
\end{tabular}

Note: n.d., not determined. 
(w/w) sucrose. Modified versions of this method developed by Mitchison and Kirschner (1986) eliminate the concentration step and directly load the cleared lysates onto a sucrose gradient, thereby minimising the loss of material. Others use an abbreviated method for rapid isolation of centrosomes by skipping the sucrose gradient step to promptly produce high yields (Blomberg-Wirschell and Doxsey, 1998). Centrosomes obtained by this method are less pure but suitable for structural and functional assays. Centrosome containing fractions from the final sucrose gradient are flash-frozen in liquid nitrogen and can be stored at $-80^{\circ} \mathrm{C}$ for several months with little effect on structure or function. Successful enrichment throughout the isolation procedure is monitored by immunofluorescence (IF) microscopy, electron microscopy and biochemical assays (sodium dodecyl sulfate-polyacrylamide gel electrophoresis (SDS-PAGE) and Western blotting). For IF, centrosomes from aliquots of subsequent isolation steps are sedimented onto cover slips in Corex tubes containing Plexiglas adapters, as described by Evans et al. (1985), followed by detection using centrosomespecific antibodies. The yield of centrosomes isolated with these methods can vary between $15 \%$ and $50 \%$ at up to 2000-fold enrichment. See also: Animal Cell Separation and Fractionation; Centrifugation Techniques; Immunofluorescence; Western Blotting: Immunoblotting

A method for isolating centrosomes from syncytial Drosophila embryos was first described by Moritz et al. (1995). Using this embryonic system as centrosome source has several advantages: multigram quantities of embryos can be obtained in a few hours; large populations of flies are maintained at relatively low costs; during rapid nuclear divisions before cellularisation, centrosomes are less likely to be attached to the nuclear membrane; and furthermore, the highly mitotic embryos contain centrosomes with large amounts of PCM compared to mammalian centrosomes that are primarily derived from interphase cells. Thirty to fifty grams of embryos are first dechorionated and disrupted mechanically with a Dounce homogeniser. The resulting slurry is filtered and centrifuged twice to remove nuclei, yolk, debris and lipids. Centrosomes in the homogenate supernatant are then enriched via density centrifugation on two consecutive sucrose step gradients. At this stage, centrosomes can be snap-frozen and stored at $-80^{\circ} \mathrm{C}$. The yield of such preparations lies around $10 \%$ with a 1000-fold enrichment as judged by the number of centrosomes per milligram in the starting embryo homogenate compared to the peak centrosome fraction. Lange et al. (2000) introduced a method in which centrosomes from enriched sucrose fractions are further purified by immunomagnetic isolation using beads that are crosslinked with affinity-purified anti- $\gamma$-tubulin antibody. This additional immunoaffinity-based isolation step leads to greatly increased purity of preparations, suitable to determine the Drosophila centrosome proteome. Enrichment, yield and intact morphology of isolated centrosomes are monitored as described earlier. An example of IF microscopic analysis of centrosomes from different isolation stages is given in Figure 1. See also: Cleavage and Gastrulation in Drosophila Embryos

\section{Applications}

All these centrosome isolation procedures have provided the basis for studying this cell organelle's morphological, functional and biochemical features in various species. Since centrioles stably maintain their paired configuration throughout the isolation, it was possible to depict their ultrastructural organisation in great detail by several electron microscopy analyses (Blackburn et al., 1978; Bornens et al., 1987; Paintrand et al., 1992; Chrétien et al., 1997). Moreover, most isolation protocols only minimally affect the microtubule nucleation capacity, which allowed detailed in vitro examination of its main function (Mitchison and Kirschner, 1984; Pereira and Schiebel, 1997; Tassin and Bornens, 1999). When incubated with exogenous fluorescently labelled tubulin and guanosine triphosphate (GTP), isolated centrosomes nucleate asters of microtubules. This experimental set-up was used to examine the effects of chemicals, proteins and antibodies on microtubule nucleation. Microinjection of human centrosomes into Xenopus eggs induces cleavage leading to parthenogenesis, thus demonstrating in vivo functionality of these preparations (Tournier et al., 1989). See also: Electron Microscopy; Xenopus as an Experimental Organism

To identify the molecular components of centrosomes, various approaches have been used. For instance, generation of antibodies led to the identification of several components of the human and Dictyostelium centrosome as well as of the yeast spindle pole body (Rout and Kilmartin, 1990; Gräf, 2001). IF microscopy using these antibodies allows the discrimination of centriolar and PCM components and furthermore the detection of cell cycle specific localisation of centrosome proteins (Lange and Gull, 1995). Another more direct approach is to electrophoretically separate isolates and apply highmass accuracy matrix-assisted laser desorption ionisation (MALDI) peptide mass mapping to the analysis of Coomassie-stained gel bands. A pioneering study using this approach in yeast led to the identification of 11 previously unknown spindle pole body components, of which localization of 8 to the centrosome could be confirmed via GFP (green florescent protein)-tagging (Wigge et al., 1998). In the following years, rapid advances in sequencing and MSbased techniques have greatly contributed in increasing our knowledge of the molecular composition of centrosomes. In a study with isolated Chlamydomonas basal bodies Keller et al. (2005) identified 53 centriole-associated proteins using multidimensional protein identification technology (MudPIT), an LC-LC-MS/MS approach with no prior electrophoretic separation. Since Chlamydomonas basal bodies possess little PCM, the authors of this study claim to have determined the first centriole proteome. Some of these components are highly conserved and have been linked to ciliopathies. See also: Green Fluorescent 


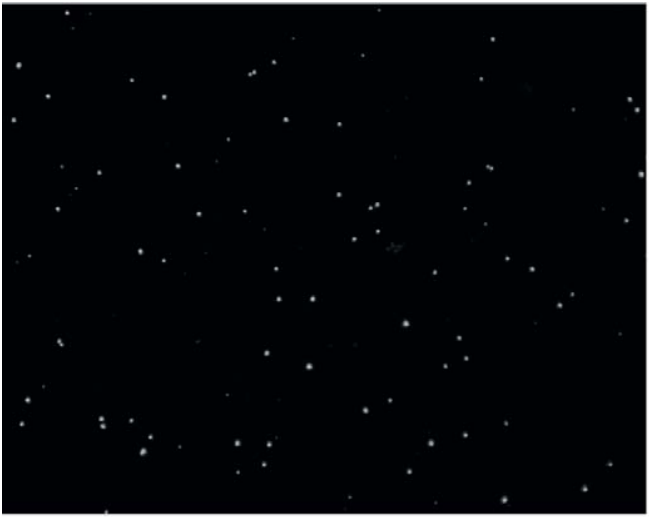

(a)

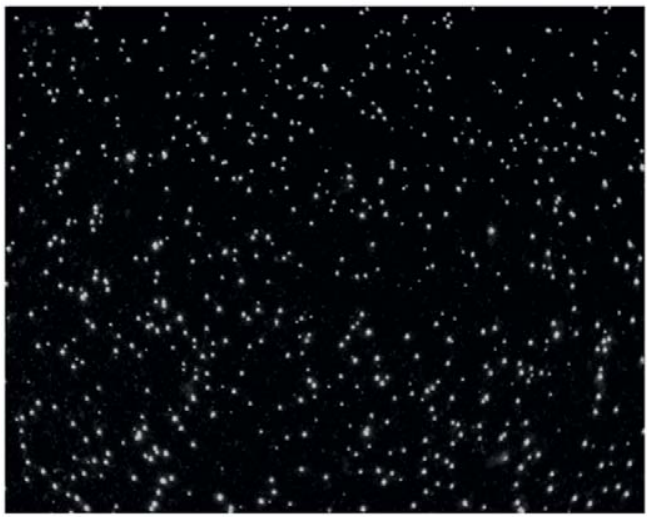

(c)

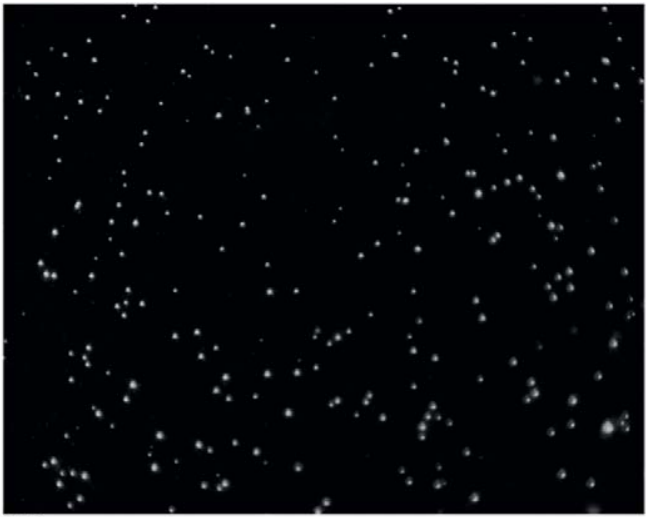

(b)

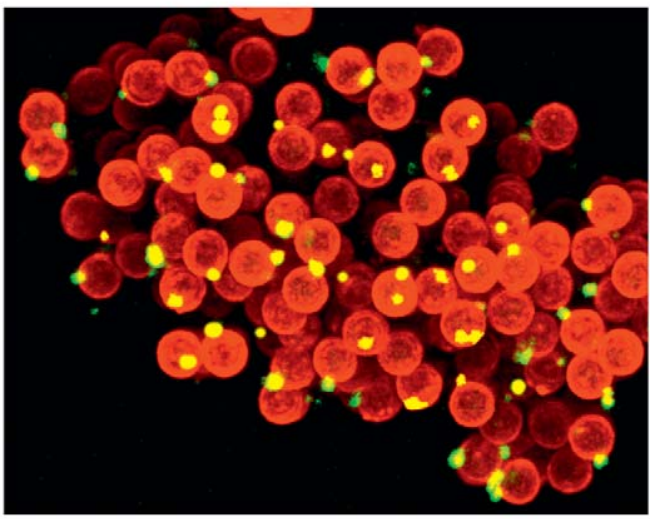

(d)

Figure 1 This image shows the consecutive isolation steps assayed by immunofluorescence microscopy using an anti- $\gamma$-tubulin antibody to evaluate number and integrity of isolated centrosomes: (a) centrosomes in embryo homogenate; (b) enriched centrosome fraction from first sucrose gradient; (c) enriched centrosome fraction from second sucrose gradient; (d) immunopurified centrosomes. Centrosomes are shown in yellow and beads are shown in red. Reproduced from Lehmann et al. (2006) with permission from Wiley.

Protein (GFP); Production of Monoclonal Antibodies; Protein Characterization in Proteomics

A third study by Andersen et al. (2003) identified 23 novel and 41 likely candidates of the human interphase centrosome by applying MS-based proteomic analysis of centrosomes isolated from KE37 (human T lymphoblastic cell line) cells in combination with protein correlation profiling, a technique that permits the analysis of multiprotein complexes, which can be enriched but not purified to homogeneity. In this method, peptides are quantified through multiple centrifugation fractions to generate a consensus protein elution profile from known centrosome components. Proteins displaying similar profiles were considered centrosomal and localisation to centrosomes was verified via expression of GFP-tagged candidates in U2OS (human osteosarcoma cell line) cells. A human centrosomal protein database, CentrosomeDB, has been established that comprises data collected from this and other studies and also contains information on homologues in other species (Nogales-Cadenas et al., 2009). It is to be expected that this data set will grow significantly in the coming years, ultimately leading to the identification of the entire centrosome proteome in various organisms.

\section{Perspectives}

Despite intense research on the biology of the centrosome during the past decades, many aspects of its functional, structural and biochemical properties still remain to be elucidated. An important tool, which has emerged over the past years, to characterise centrosome functions is RNAi. In combination with high-throughput microscopy assays, this method allows the integration of centrosomal proteins into cellular signalling pathways and thereby has the potential of revealing previously unknown functions of the centrosome. Genome-wide or targeted RNAi screens have already been used to unravel the molecular pathways of centrosome biogenesis, duplication, maturation and separation, spindle assembly and cell cycle progression and to identify novel proteins with centrosome-related functions (Goshima et al., 2007; Dobbelaere et al., 2008).

Regarding structural features, in vitro approaches based on electron microscopy of isolated centrosomes have for a long time been used to obtain detailed information on the configuration of centrosomes and centrioles, respectively. Technical advances in this field, in particular the development of cryo-electron tomography, have opened the path to 
visualise the spatial organisation of the entire proteome of an organelle with a resolution that is sufficient to depict individual molecules. Internal structures like the saltinsoluble scaffold of the centrosome, termed centromatrix, have been discovered via electron microscopy (Schnackenberg and Palazzo, 1999). It will be informative to learn about the supramolecular organisation of this and other centrosomal subcomplexes in the future. A recent study, in which laser manipulation of individual organelles was used to reveal its charge and hydrodynamic behaviour, has shown that isolated centrosomes are also suitable for investigating their biophysical properties (Hormeno et al., 2009). See also: Electron Cryomicroscopy and Three-dimensional Computer Reconstruction of Biological Molecules

Our knowledge of the centrosome's molecular inventory has continuously increased during the past decades, mainly owing to rapid improvement of MS-based techniques. A future goal will be to reduce the number of falsely identified proteins, which can be achieved by improved purity of preparations through affinity isolation procedures that have so far only been applied to Drosophila centrosomes. Another major goal will be to study the dynamics of the centrosomal proteome regarding composition and posttranslational modifications in the context of cell cycle progression and differentiation or as response to drug treatment or depletion of signalling components. A powerful tool to study these aspects became available with the introduction of quantitative mass spectrometry in combination with isotope labelling (Hilger et al., 2009). Moreover, deciphering the differences of the molecular inventory of this organelle in normal and cancer cells, in the latter of which centrosomal proteins are frequently deregulated, will lead to a better understanding of the mechanisms of this disease. See also: Quantitative Proteomics $\left(\right.$ ICAT $\left.^{\mathrm{TM}}\right)$

\section{References}

Andersen JS, Wilkinson CJ, Mayor T et al. (2003) Proteomic characterization of the human centrosome by protein correlation profiling. Nature 426: 570-574.

Blackburn GR, Barrau MD and Dewey WC (1978) Partial purification of centrosomes from Chinese hamster ovary cells. Experimental Cell Research 113: 183-187.

Blomberg-Wirschell M and Doxsey SJ (1998) Rapid isolation of centrosomes. Methods in Enzymology 298: 228-238.

Bornens M and Moudjou M (1999) Studying the composition and function of centrosomes in vertebrates. Methods in Cell Biology 61: $13-34$.

Bornens M, Paintrand M, Berges J, Marty MC and Karsenti E (1987) Structural and chemical characterization of isolated centrosomes. Cell Motility and the Cytoskeleton 8: 238-249.

Chrétien D, Buendia B, Fuller SD and Karsenti E (1997) Reconstruction of the centrosome cycle from cryoelectron micrographs. Journal of Structural Biology 120: 117-133.

Dobbelaere J, Josue F, Suijkerbuijk S et al. (2008) A genome-wide RNAi screen to dissect centriole duplication and centrosome maturation in Drosophila. PLoS Biology 6: 1975-1990.
Dutcher SK (1995) Purification of basal bodies and basal body complexes from Chlamydomonas reinhardtii. Methods in Cell Biology 47: 323-334.

Evans L, Mitchison T and Kirschner M (1985) Influence of the centrosome on the structure of nucleated microtubules. Journal of Cell Biology 100: 1185-1191.

Goshima G, Wollman R, Goodwin SS et al. (2007) Genes required for mitotic spindle assembly in Drosophila $\mathrm{S} 2$ cells. Science $\mathbf{3 1 6}$ : 417-421.

Gosti-Testu F, Marty MC, Berges J, Maunoury R and Bornens M (1986) Identification of centrosomal proteins in a human lymphoblastic cell line. EMBO Journal 5: 2545-2550.

Gräf R (2001) Isolation of centrosomes from Dictyostelium. Methods in Cell Biology 67: 337-357.

Hilger M, Bonaldi T, Gnad F and Mann M (2009) Systems-wide analysis of a phosphatase knock-down by quantitative proteomics and phosphoproteomics. Molecular and Cellular Proteomics 8: 1908-1920.

Hormeno S, Ibarra B, Chichon FJ et al. (2009) Single centrosome manipulation reveals its electric charge and associated dynamic structure. Biophysical Journal 97: 1022-1030.

Keller LC and Marshall WF (2008) Isolation and proteomic analysis of Chlamydomonas centrioles. Methods in Molecular Biology 432: 289-300.

Keller LC, Romijn EP, Zamora I, Yates JR 3rd and Marshall WF (2005) Proteomic analysis of isolated Chlamydomonas centrioles reveals orthologs of ciliary-disease genes. Current Biology 15: $1090-1098$.

Kellogg DR, Moritz M and Alberts BM (1994) The centrosome and cellular organization. Annual Review of Biochemistry 63: 639-674.

Komesli S, Tournier F, Paintrand M et al. (1989) Mass isolation of calf thymus centrosomes: identification of a specific configuration. Journal of Cell Biology 109: 2869-2878.

Lange BM and Gull K (1995) A molecular marker for centriole maturation in the mammalian cell cycle. Journal of Cell Biology 130: 919-927.

Lange BM, Bachi A, Wilm M and Gonzalez C (2000) Hsp90 is a core centrosomal component and is required at different stages of the centrosome cycle in Drosophila and vertebrates. EMBO Journal 19: 1252-1262.

Lehmann V, Müller H and Lange BM (2006) Immunoisolation of centrosomes from Drosophila melanogaster. Current Protocols in Cell Biology 29: 3.17.1-3.17.13 (chap. 3: unit 3).

Mitchison TJ and Kirschner MW (1984) Microtubule assembly nucleated by isolated centrosomes. Nature 312: 232-237.

Mitchison TJ and Kirschner MW (1986) Isolation of mammalian centrosomes. Methods in Enzymology 134: 261-268.

Moritz M and Alberts BM (1999) Isolation of centrosomes from Drosophila embryos. Methods in Cell Biology 61: 1-12.

Moritz M, Braunfeld MB, Fung JC et al. (1995) Threedimensional structural characterization of centrosomes from early Drosophila embryos. Journal of Cell Biology 130: 1149-1159.

Nogales-Cadenas R, Abascal F, Diez-Perez J, Carazo JM and Pascual-Montano A (2009) CentrosomeDB: a human centrosomal proteins database. Nucleic Acids Research 37: D175-D180.

Paintrand M, Moudjou M, Delacroix H and Bornens M (1992) Centrosome organization and centriole architecture: their 
sensitivity to divalent cations. Journal of Structural Biology $\mathbf{1 0 8}$ : 107-128.

Palazzo RE and Vogel JM (1999) Isolation of centrosomes from Spisula solidissima oocytes. Methods in Cell Biology 61: 35-56.

Pereira G and Schiebel E (1997) Centrosome-microtubule nucleation. Journal of Cell Science 110(part 3): 295-300.

Roobol A, Havercroft JC and Gull K (1982) Microtubule nucleation by the isolated microtubule-organizing centre of Physarum polycephalum myxamoebae. Journal of Cell Science $\mathbf{5 5}$ : 365-381.

Rout MP and Kilmartin JV (1990) Components of the yeast spindle and spindle pole body. Journal of Cell Biology 111: 1913-1927.

Schnackenberg BJ and Palazzo RE (1999) Identification and function of the centrosome centromatrix. Biology of the Cell 91: 429-438.

Tassin AM and Bornens M (1999) Centrosome structure and microtubule nucleation in animal cells. Biology of the Cell 91: 343-354.

Thompson-Coffe C, Coffe G, Schatten H, Mazia D and Schatten G (1996) Cold-treated centrosome: isolation of centrosomes from mitotic sea urchin eggs, production of an anticentrosomal antibody, and novel ultrastructural imaging. Cell Motility and the Cytoskeleton 33: 197-207.

Tournier F, Karsenti E and Bornens M (1989) Parthenogenesis in Xenopus eggs injected with centrosomes from synchronized human lymphoid cells. Developmental Biology 136: 321-329.

Wigge PA, Jensen ON, Holmes S et al. (1998) Analysis of the Saccharomyces spindle pole by matrix-assisted laser desorption/ionization (MALDI) mass spectrometry. Journal of Cell Biology 141: 967-977.

\section{Further Reading}

Andersen JS and Mann M (2006) Organellar proteomics: turning inventories into insights. EMBO Reports 7: 874-879.

Badano JL, Teslovich TM and Katsanis N (2005) The centrosome in human genetic disease. Nature Reviews. Genetics 6: 194-205.

Bettencourt-Dias M and Glover DM (2007) Centrosome biogenesis and function: centrosomics brings new understanding. Nature Reviews. Molecular Cell Biology 8: 451-463.

Doxsey S (2001) Re-evaluating centrosome function. Nature Reviews. Molecular Cell Biology 2: 688-698.

Gonzalez C (2008) Centrosome function during stem cell division: the devil is in the details. Current Opinion in Cell Biology 20: 694-698.

Lange BM (2002) Integration of the centrosome in cell cycle control, stress response and signal transduction pathways. Current Opinion in Cell Biology 14: 35-43.

Leis A, Rockel B, Andrees L and Baumeister W (2009) Visualizing cells at the nanoscale. Trends in Biochemical Sciences 34: 60-70.

Nigg EA (ed.) (2005) Centrosomes in Development and Disease. Wiley-VCH: Weinheim.

Palazzo RE and Davis TN (eds) (2001) Centrosomes and spindle pole bodies. Methods in Cell Biology, vol. 67. San Diego: Academic Press.

Pelletier L, O’Toole E, Schwager A, Hyman AA and MüllerReichert T (2006) Centriole assembly in Caenorhabditis elegans. Nature 444: 619-623. 